\title{
Identify practice gaps in medication education through surveys to patients and physicians
}

\author{
This article was published in the following Dove Press journal: \\ Patient Preference and Adherence \\ 9 October 2015 \\ Number of times this article has been viewed
}

\author{
Zhan-Miao $\mathrm{Yi}^{1}$ \\ Xiao-Jie Zhi' ${ }^{2}$ \\ Ling Yang ${ }^{3}$ \\ Shu-Sen Sun ${ }^{4}$ \\ Zhuo Zhang ${ }^{2}$ \\ Zhi-Ming Sun ${ }^{2}$ \\ Suo-Di Zhai' \\ 'Department of Pharmacy, Peking \\ University Third Hospital, Beijing, \\ People's Republic of China; ${ }^{2}$ School \\ of Pharmaceutical Science, ${ }^{3}$ School \\ of Public Health, Peking University, \\ Beijing, People's Republic of China; \\ ${ }^{4}$ College of Pharmacy, Western \\ New England University, Springfield, \\ MA, USA
}

Background: Effective communication and education formats between health care providers and patients about medication use are associated with patients' satisfaction, recall of information, and eventually their health status. Limited research exists on physician-delivered education interventions, as well as on whether the current content of medication education and delivery formats satisfies the needs of both patients and physicians. Our objective was to identify the practice gaps regarding medication education content and delivery.

Methods: Separate surveys were obtained from ambulatory care patients presenting to the outpatient pharmacy for medication pickups, and physicians working at the hospital clinics.

Results: A total of 108 patients completed the patient survey, and 116 hospital clinic physicians completed the physician survey. Female patients had a higher degree of concern regarding medication information compared with male patients ( $4.04 \pm 0.65$ versus $3.58 \pm 0.66, P=0.001$ ). Physicians were less likely to educate patients regarding their medications' on drug-drug interactions (24.3\%), drug-food interactions (24.3\%), and what to do about their prescriptions if an adverse reaction is experienced (24.3\%) during physician-patient encounters. Patients' most desired education format was physician counseling $(82.4 \%)$ and the second most desired education format was pharmacist counseling (50.9\%). Medication device demonstration $(7.0 \%)$ was the least used educational format delivered to patients by physicians, and patients would like to see an increased education delivery format through medication device demonstration (Method desired $[\mathrm{MD}]$ - Method received $[\mathrm{MR}]=12.0 \%$ ). Patients would like to see expanded roles of patient focused handout (MD-MR=22.2\%), telephone consultation $(21.2 \%)$, pharmacist counseling (12.9\%), the use of medication database embedded within the hospital information system (12.2\%) and device demonstration (12.0\%).

Conclusion: This study illustrates that there are practice gaps in current medication education both in terms of content and delivery format. The study provided valuable information in designing and implementing future education activities that are drivers of good medication use and adherence. Keywords: medication education, medication adherence, degree of concern, education content, education format

\section{Introduction}

Medication information and counseling are an essential part of pharmaceutical care, ${ }^{1}$ especially during patient-provider interactions at hospital clinic visits and outpatient pharmacy medication dispensing. Medication education usually takes the form of verbal and/or written formats. Written consumer medication information (CMI) is considered an important component of patient education internationally in promotion of safe and effective medicine-taking behavior. ${ }^{2}$ Other effective forms of written education have been explored such as patient-focused handouts, use of pictorial aids and plain language, to overcome the limitations of CMI.,4 Verbal communication is equally important, especially for patients who have barriers to reading printed materials.
Correspondence: Suo-Di Zhai Department of Pharmacy, Peking University Third Hospital, No 49 North Garden Road, Haidian District, Beijing I00I9I, People's Republic of China Email zhaisuodi@I63.com 
The most often expressed barrier to providing effective verbal education is lack of contact time between patients and health care providers. ${ }^{5}$

For education to be effective, providers should consider delivering patient-centered medication education based on patient's needs and through formats supported by patients and providers. For ambulatory clinic hospital patients in People's Republic of China, clinic physicians usually serve as providers for medication education to patients. Pharmacists working at hospital outpatient pharmacies can provide additional medication consultation upon patients' requests. There is limited research on physician-delivered education interventions, ${ }^{6}$ as well as research on whether the current content of medication education and delivery formats satisfy the needs of both patients and physicians. Based on our observations, we believe there is a mismatch between the current practice and the preferred practice in medication education. The aim of this study was to identify the practice gaps. The specific objectives of this study were to determine 1) the provider and patients' degree of concern regarding components of medication information, 2) the contents of medication education received by patients compared with those delivered by providers and to identify where gaps exist, and 3) the formats of medication education received by patients compared with those delivered by providers and to identify where gaps exist. This study is warranted in order to provide patient-centered education with engagements from both patients and health care providers.

\section{Methods}

\section{Setting and participants}

Our study took place at the Peking University Third Hospital, a tertiary academic-teaching hospital in the city of Beijing. The survey questionnaires to patients and to physicians were developed by the research team based on the typical components of CMI and patient medication consultation records. The paper-based questionnaire was structured according to the study objectives. Each survey contained four parts designed to collect information on participant demographics, degree of concern regarding medication information, education content, and delivery format of medication information. Demographic information was obtained for the patient sample on sex, age, education level, and method of medication payment. For the physician sample, demographic information was collected on sex, age, specialty, and level of professional rank. There were 35 questions in the patient survey and 38 questions on the physician survey. Both surveys were designed to take no more than 30 minutes to complete.

Presurveys were conducted before the pilot to assess the validity and/or reliability of the survey instruments. A total of 20 questionnaires were sent with 17 completed. Final survey questions were assessed and modified based on the analysis of the results of the presurveys.

The patient study population was ambulatory care patients presenting to the outpatient pharmacy for medication pickups. The physician surveys were sent to a group of physicians working at the hospital clinics with considerations of clinical specialty-internal medicine versus noninternal medicine and levels of professional ranks, etc. Inclusion criteria for these studies were age greater than 16 years, and able to complete the questionnaire with little or no help from study investigators.

\section{Data collection and analysis}

All collected data were coded and entered into a database in the SPSS (version 20.0, IBM Corporation, Armonk, NY, USA, 2011). Cronbach's $\alpha$ was adopted for the measurement of the reliability of the questionnaire. Descriptive statistics, a Student's $t$-test and $\chi^{2}$ test for question items were computed. The level of statistical significance was set at $P<0.05$ (two-tailed analysis).

\section{Ethical approval}

The study protocol was approved by the Peking University Third Hospital Ethics Committee.

\section{Results}

\section{Participants' demographics}

A total of 123 patients were invited to participate, of which 120 consented (97.6\% response), and 108 questionnaires were completed (90\% completion). Patients completed the survey in person at pharmacy pickups. It took study investigators 2 hours per day to complete the survey in a week. The majority of study participants was young and middle-aged with an education level at or above college. There were no statistically significant differences among study population regarding sex, age, education level, or method of payment. Demographic details of participants are shown in Table 1.

A total of 127 paper-based surveys were sent to the hospital clinic physicians by hospital pharmacists, 118 were returned (92.9\% response), and 116 completed all sections (91.3\% completion). The survey was open for 1 month. The majority of study participants were between the ages of 20 and 40 years old with approximately one-third of the 
Table I Demographic characteristics of patients $(\mathrm{N}=108)$

\begin{tabular}{ll}
\hline Demographics & Participants, $\mathbf{n}(\%)$ \\
\hline Sex & $47(43.5)$ \\
Male & $61(56.5)$ \\
Female & \\
Age (years) & $4(3.7)$ \\
$<20$ & $40(37.0)$ \\
$20-30$ & $28(25.9)$ \\
$30-40$ & $19(17.6)$ \\
$40-50$ & $10(9.3)$ \\
$50-60$ & $7(6.5)$ \\
$>60$ & \\
Education level & $7(6.5)$ \\
Less than high school & $10(9.3)$ \\
High school & $25(23.1)$ \\
2-year college & $46(42.6)$ \\
$4-y e a r$ college & $20(18.5)$ \\
Above college & \\
Method of medication payment & $20(18.5)$ \\
Self-pay & $37(34.3)$ \\
Free medical care & $18(16.7)$ \\
Insurance for urban residents & $29(26.9)$ \\
Basic insurance for urban workers & $2(1.9)$ \\
New rural cooperative medical system & $2(1.9)$ \\
Other type of insurance &
\end{tabular}

physicians being internists. There were no statistically significant differences among study population regarding sex, age, specialty, or levels of professional rank. Demographic details of participants are shown in Table 2.

\section{Degree of concern regarding components of medication information}

Both patient's and physician's degree of concern regarding different components of medication information were

Table 2 Demographic characteristics of physicians ( $\mathrm{N}=\mathrm{I} \mid 6)$

\begin{tabular}{ll}
\hline Demographics & Participants, $\mathbf{n}$ (\%) \\
\hline Sex & \\
$\quad$ Male & $60(51.7)$ \\
Female & $56(48.3)$ \\
Age (years) & \\
$<20$ & $2(1.7)$ \\
$20-30$ & $33(28.4)$ \\
$30-40$ & $59(50.9)$ \\
$40-50$ & $18(15.5)$ \\
$50-60$ & $2(1.7)$ \\
$>60$ & $2(1.7)$ \\
Specialty & \\
Internal medicine & $44(37.9)$ \\
Surgery & $72(62.1)$ \\
Level of professional rank & \\
Entry-level & $38(32.8)$ \\
Mid-level & $46(39.7)$ \\
Advanced level & $32(27.6)$ \\
\hline
\end{tabular}

assessed, and the results are shown separately in Tables 3 and 4 . For the quantitative assessment, each item was rated with a 5-point Likert scale (ranging from 1= "never care" to $5=$ "care a lot"). For patients, the top five categories with the highest degree of concern in descending order were dose (4.40), administration route (4.23), duration of therapy (4.16), administration instructions (4.06), and dosing frequency (4.03). The five categories with the least degree of concern in ascending order were medication price, drug storage, medication name, drug-food interactions, and medication efficacy. For physicians, the top five categories with the highest degree of concern in descending order were indications (4.45), administration route (4.42), dose (4.35), dosing frequency (4.28), and duration of therapy (4.12). The five categories with the least degree of concern in ascending order were medication storage, what to do if a dose is missed, interactions or contraindications, what to do if no response from medication and onset of action.

The overall mean score of average degree of concern regarding medication information based on patient demographics is presented in Table 5. There were no statistically significant differences in terms of age, education level, and method of payment. However, when calculated for sex, there was a statistically significant difference (female $4.04 \pm 0.65$ versus male $3.58 \pm 0.66, P=0.001$ ).

The overall mean score of average degree of concern regarding medication information based on physician demographics is presented in Table 6. The demographic categories of age and sex showed no statistically significant differences. However, there were statistically significant differences in terms of medical specialty (internal medicine $4.12 \pm 0.44$ versus surgery $3.78 \pm 0.81, P=0.004)$, and levels of professional ranks (entry-level $3.68 \pm 0.71$ versus mid-level $4.02 \pm 0.61$ versus advanced-level $4.01 \pm 0.79, P=0.048$ ).

\section{Content of medication education provided to patients by physicians}

Content of medication education provided by physicians were assessed through physician survey. Physicians were more likely to provide education to patients about their medications regarding dose $(88.7 \%)$, dosing frequency $(80.9 \%)$, indications $(80.0 \%)$, administration route $(79.1 \%)$, adverse reactions $(70.4 \%)$, medication name $(67.5 \%)$, and duration of therapy (59.1\%). However, information on drug storage $(21.9 \%)$, what to do if adverse reaction was experienced (24.3\%), drug-drug interactions (24.3\%), drug-food interactions (24.3\%), contraindications (39.1\%), and onset of action $(43.1 \%)$ were less likely to be provided by physicians. 
Table 3 Patient degree of concern regarding medication information

\begin{tabular}{|c|c|c|c|c|c|c|}
\hline \multirow[t]{2}{*}{ Items } & \multicolumn{5}{|l|}{ n (\%) } & \multirow[t]{2}{*}{ Mean ranking } \\
\hline & Never care & Seldom care & Sometimes care & Often care & Care a lot & \\
\hline Dose & $0(0.0)$ & $7(6.5)$ & $7(6.5)$ & $30(27.8)$ & $64(59.3)$ & 4.40 \\
\hline Administration route & $3(2.8)$ & $7(6.5)$ & $10(9.3)$ & $30(27.8)$ & $58(53.7)$ & 4.23 \\
\hline Duration of therapy & I (0.9) & $8(7.4)$ & $12(11.1)$ & $39(36.1)$ & $48(44.4)$ & 4.16 \\
\hline Administration instructions & $0(0.0)$ & $10(9.3)$ & $16(14.8)$ & $40(37.0)$ & $42(38.9)$ & 4.06 \\
\hline Dosing frequency & $2(1.9)$ & $6(5.6)$ & $21(19.4)$ & $37(34.3)$ & $42(38.9)$ & 4.03 \\
\hline Adverse reactions & $\mathrm{I}(0.9)$ & $13(12.0)$ & $19(17.6)$ & $31(28.7)$ & 44 (40.7) & 3.96 \\
\hline Indication(s) & I (0.9) & $5(4.6)$ & $27(25.0)$ & $4 I(38.0)$ & $34(31.5)$ & 3.94 \\
\hline Drug-drug interactions & $3(2.8)$ & $14(13.0)$ & $23(21.3)$ & $26(24.1)$ & $42(38.9)$ & 3.83 \\
\hline Onset of action & $0(0.0)$ & $15(13.9)$ & $24(22.2)$ & $35(32.4)$ & $34(3 \mid .5)$ & 3.81 \\
\hline Special precautions & $2(1.9)$ & II (I0.2) & $30(27.8)$ & $32(29.6)$ & $33(30.6)$ & 3.77 \\
\hline Drug-disease interactions & $6(5.6)$ & $15(13.9)$ & $23(2 \mid .2)$ & $27(25.0)$ & $37(34.3)$ & 3.69 \\
\hline Medication efficacy & $4(3.7)$ & $21(19.4)$ & $18(16.7)$ & $29(26.9)$ & $36(33.3)$ & 3.67 \\
\hline Drug-food interactions & $4(3.7)$ & $19(17.6)$ & $25(23.1)$ & $25(23.1)$ & $35(32.4)$ & 3.63 \\
\hline Medication name & $8(7.4)$ & $13(12.0)$ & $25(23.1)$ & $32(29.6)$ & $30(27.8)$ & 3.58 \\
\hline Drug storage & $6(5.6)$ & $18(16.7)$ & $22(20.4)$ & $37(34.3)$ & $25(23.1)$ & 3.53 \\
\hline Medication price & $13(12.0)$ & $23(21.3)$ & $26(24.1)$ & $30(27.8)$ & $16(14.8)$ & 3.12 \\
\hline
\end{tabular}

Note: "Each item was rated with a 5-point Likert scale (ranging from I= "never care" to $5=$ "care a lot").

\section{Content of medication education received by patients and if this matched patients' desires}

The content of medication education reported by patients versus what the patient actually desired to receive was assessed, and the results are shown in Table 7. Patients were more likely to be educated on medication indication, medication name, dose, administration route, adverse reactions, dose frequency, and contraindications of their prescribed medications. However, patients were less likely to be educated on what to do if adverse reactions occur, medication storage, and drug-drug and/or drug-food interactions. The items patients most desired to receive during verbal education were adverse reactions, indication, drug-drug interactions, drug-food interactions, contraindications, dosing, and what to do if adverse reactions occur. The least desired items patients wanted to be educated on were medication name, medication storage, and administration route.

\section{Format of medication education delivered to patients by physicians}

The survey revealed that the following formats were frequently used by physicians to provide medication education to patients: physician counseling (77.4\%), CMI (46.2\%), medication database embedded within hospital information system (33.0\%), auxiliary labels (25.2\%), and lectures

Table 4 Physician degree of concern regarding medication information

\begin{tabular}{|c|c|c|c|c|c|c|}
\hline \multirow[t]{2}{*}{ Items } & \multicolumn{5}{|l|}{ n (\%) } & \multirow[t]{2}{*}{ Mean ranking } \\
\hline & Never care & Seldom care & Sometimes care & Often care & Care a lot & \\
\hline Indication(s) & $2(1.7)$ & I (0.9) & $6(5.2)$ & 41 (35.3) & $66(56.9)$ & 4.45 \\
\hline Administration route & $2(1.7)$ & $0(0.0)$ & $10(8.6)$ & $39(33.6)$ & $65(56.0)$ & 4.42 \\
\hline Dose & $2(1.7)$ & $0(0.0)$ & $12(10.3)$ & $43(37.1)$ & $59(50.9)$ & 4.35 \\
\hline Dosing frequency & $2(1.7)$ & $0(0.0)$ & $13(\mid 1.2)$ & $50(43.1)$ & $5 I(44.0)$ & 4.28 \\
\hline Duration of therapy & $3(2.6)$ & $2(1.7)$ & $14(12.1)$ & $56(48.3)$ & $4 I(35.3)$ & 4.12 \\
\hline Adverse reactions & $2(1.7)$ & $7(6.0)$ & $17(14.7)$ & $40(34.5)$ & $50(43.1)$ & 4.11 \\
\hline Medication name & $4(3.4)$ & $5(4.3)$ & $28(24.1)$ & $37(31.9)$ & $42(36.2)$ & 3.93 \\
\hline Onset of action & $3(2.6)$ & $13(1 \mid .2)$ & $27(23.3)$ & $41(35.3)$ & $32(27.6)$ & 3.74 \\
\hline What to do if no anticipated effects & $3(2.6)$ & $17(14.7)$ & $27(23.3)$ & $4 \mid(35.3)$ & $28(24.1)$ & 3.64 \\
\hline $\begin{array}{l}\text { Drug-drug/drug-food/drug-disease } \\
\text { interactions/contraindications }\end{array}$ & $2(1.7)$ & $17(14.7)$ & $28(24.1)$ & $46(39.7)$ & $23(19.8)$ & 3.61 \\
\hline What to do if a dose is missed & $7(6.0)$ & $28(24.1)$ & $36(31.0)$ & $33(28.4)$ & $12(10.3)$ & 3.13 \\
\hline Drug storage & $4(3.4)$ & $34(29.3)$ & $39(33.6)$ & $25(21.6)$ & $14(12.1)$ & 3.09 \\
\hline
\end{tabular}

Note: aEach item was rated with a 5 -point Likert scale (ranging from I= "never care" to $5=$ "care a lot"). 
Table 5 Patient average degree of concern based on demographic category

\begin{tabular}{lll}
\hline Demographics & $\begin{array}{l}\text { Average degree of concern } \\
\text { regarding medication } \\
\text { information (mean } \pm \text { SD) }\end{array}$ & \\
\hline Sex & & 0.001 \\
Male & $4.04 \pm 0.65$ & \\
Female & $3.58 \pm 0.66$ & 0.227 \\
Age (years) & & \\
$<20$ & $3.58 \pm 0.94$ & \\
20-30 & $3.74 \pm 0.69$ & \\
30-40 & $3.77 \pm 0.78$ & \\
40-50 & $4.06 \pm 0.53$ & \\
50-60 & $4.19 \pm 0.52$ & \\
>60 & $3.64 \pm 0.70$ & \\
Education level & & \\
Less than high school & $3.57 \pm 0.49$ & \\
High school & $4.01 \pm 0.50$ & \\
2-year college & $4.11 \pm 0.71$ & \\
4-year college & $3.73 \pm 0.65$ & \\
Above college & $3.74 \pm 0.82$ & \\
Method of medication & & \\
payment & 3.660 \\
Self-pay & $3.92 \pm 0.73$ & \\
Free medical care & $3.93 \pm 0.68$ & \\
Insurance for urban & & \\
residents & $3.84 \pm 0.53$ & \\
Basic insurance for & & \\
urban workers & & \\
New rural cooperative & $3.63 \pm 0.18$ & \\
medical system & & \\
Other type of & $2.84 \pm 0.40$ & \\
insurance & & \\
\hline
\end{tabular}

Table 6 Physician average degree of concern based on demographic category

\begin{tabular}{llc}
\hline Demographics & \multicolumn{1}{l}{$\begin{array}{l}\text { Average degree of concern regarding } \\
\text { medication information (mean } \pm \text { SD) }\end{array}$} & $P$-value \\
\hline Sex & & 0.662 \\
$\quad$ Male & $3.87 \pm 0.82$ & \\
$\quad$ Female & $3.93 \pm 0.58$ & 0.216 \\
Age (years) & & \\
$\quad<20$ & $3.67 \pm 1.53$ & \\
$20-30$ & $3.67 \pm 0.69$ & \\
$30-40$ & $3.97 \pm 0.67$ & \\
$40-50$ & $4.17 \pm 0.73$ & \\
$50-60$ & $4.12 \pm 0.53$ & \\
$>60$ & $3.96 \pm 1.24$ & \\
Specialty & & \\
Internal & $4.12 \pm 0.44$ & \\
medicine & & \\
Surgery & $3.78 \pm 0.81$ & \\
Professional rank & & \\
Entry-level & $3.68 \pm 0.71$ & \\
Mid-level & $4.02 \pm 0.61$ & \\
Advanced- & $4.01 \pm 0.79$ & \\
level & & \\
\hline
\end{tabular}

(23.7\%). The formats with the least frequent use were medication device demonstration $(7.0 \%)$, online $(7.8 \%)$ and telephone consultation (9.6\%), pamphlets (10.4\%), and patient-focused handout (14.8\%). Some physicians $(22.6 \%)$ also referred patients to pharmacist counseling.

\section{Format of medication education received by patients and if this matched patients' desires}

The format of medication education delivered to the patient versus the patient's desired delivery format were evaluated, and the results are shown in Table 8. Patients were more likely to be educated using the following formats: physician counseling, CMI, auxiliary labels, online consultation, and pharmacist counseling. Patients were less likely to be educated through telephone consultation and medication device demonstration. The delivery formats most desired by patients for education were physician counseling, pharmacist counseling, CMI, auxiliary label, and patient-focused handout. Patients would also like to see a decrease use of CMI but an increased use of telephone consultation, medication device demonstration, and medication database embedded within hospital information system.

\section{Discussion}

This study investigated the current practice and the practice gap in medication education through surveys to patients and physicians. The study revealed that female patients had a higher degree of concern about their medications than male patients. Male sex has been identified as one of the risk factors to low adherence to medication therapy. ${ }^{7,8}$ This sex difference suggests that when educating male patients, providers might want to find ways to better engage this patient population. For physicians, internists had higher degree of concern on medication information than surgical physicians. This may be related to more prescriptions that were prescribed by internists. Data also shows that mid-level and advancedlevel physicians had higher degree of concern on medication information than entry-level physicians.

The patient survey found that during verbal counseling sessions there was a mismatch between the information provided in comparison with the information desired by the patient. The greatest demand not being met with current counseling, as demonstrated by the difference between education provided and education desired is drug-drug interactions, drug-food interactions, and what to do if an adverse reaction experienced. It is important to note that physicians are less likely to provide those three patient desired topics during 
Table 7 Information regarding the content of medication education reported by patients

\begin{tabular}{llll}
\hline Education content & Education received (ER, \%) & Education desired (ED, \%) & Difference (ED-ER, \%) \\
\hline Adverse reactions & 49.1 & 62.0 & 12.9 \\
Indication(s) & 73.1 & 61.1 & -12.0 \\
Drug-drug interactions & 22.2 & 48.1 & 25.9 \\
Drug-food interactions & 19.4 & 45.4 & 26.0 \\
Contraindications & 45.4 & 45.4 & 0.0 \\
Dose & 59.3 & 43.5 & -15.8 \\
What to do if adverse reaction experienced & 14.8 & 43.5 & 28.7 \\
Onset of action & 28.7 & 41.7 & 13.0 \\
Duration of therapy & 27.8 & 40.7 & 12.9 \\
Dosing frequency & 46.3 & 35.2 & -11.1 \\
Administration route & 51.9 & 31.5 & -20.4 \\
Medication name & 68.5 & 26.9 & -41.6 \\
Medication storage & 19.4 & 26.9 & 7.5 \\
\hline
\end{tabular}

verbal counseling sessions as reported by physicians in this study. This is an area that pharmacists, as drug-information experts, will be able to fill in the practice gap, and be ready to provide the related information to both providers and patients. Often there is limited time available for physicians to conduct medication counseling during patients' clinic visit especially in busy hospital clinics. Research shows that tailored communication during patient education contributes to health outcomes such as information recall and medication adherence. ${ }^{9}$ When conducting medication education, health care providers should explore ways to address patients' educational needs. However, we should also keep in mind the difference between practical significance versus statistical significance. Although information such as medication name, administration route, dose, and indication were in the bottom tier of desired education from patients' perspectives, this should be provided to patients to ensure the safe use of medications. In practice, however, patients' knowledge regarding this basic information can be quickly evaluated leaving time for more complex topics. The basic topics, which are also of concern to patients, can be briefly touched on with written information provided as reinforcement. Providers can then focus verbal counseling on other topics not as easily explained and understood in written information. This will help filling the current practice gap in patient education needs identified in this study.

Currently, the most common method of medication education delivery format to our hospital clinic patients is through physician counseling. Pharmacist medication counseling is the second most desired form of education format after physician counseling through the patient survey. This suggests that patients highly value the medication education provided by pharmacists. From physician survey, it is clear that physicians also make patient referrals for pharmacist counseling. This is encouraging news for pharmacists, and pharmacists should work with physicians to coordinate patients' medication education. The outpatient pharmacy is an excellent second patient-provider encounter to provide medication education and to address patient educational needs.

Table 8 Information regarding sources of medication education reported by patients

\begin{tabular}{llll}
\hline Education delivery method & Method received (MR, \%) & Method desired (MD, \%) & Difference (MD-MR, \%) \\
\hline Physician counseling & 78.7 & 82.4 & 3.7 \\
Pharmacist counseling & 38.0 & 50.9 & 12.9 \\
CMI & 74.1 & 42.6 & -31.5 \\
Auxiliary label & 46.3 & 38.0 & -8.3 \\
Patient focused handout & 10.2 & 32.4 & 22.2 \\
Online consultation & 44.4 & 27.8 & -16.6 \\
Telephone consultation & 1.9 & 23.1 & 21.2 \\
Medication database embedded & 4.5 & 16.7 & 12.2 \\
within hospital information system & & & 12.0 \\
Device demonstration & 3.7 & 15.7 & 4.6 \\
Lectures & 11.1 & 15.7 & 4.6 \\
Pamphlets & 9.3 & 13.9 &
\end{tabular}

Abbreviation: $\mathrm{CMI}$, consumer medication information. 
The greatest demand in terms of method of education delivery to patients not being met, as demonstrated by the difference between method provided and method desired, is use of patient-focused handouts and telephone consultation. There is also an unmet demand for medication device demonstration from patients' perspectives. A study on asthma patients indicates that patients fail to comply with their asthma regimen for a wide variety of reasons, but incorrect use of inhaler devices is among the most common. ${ }^{10}$ Our physician survey shows that medication device demonstration was the least used educational format delivered to patients by physicians. It is important for pharmacists to understand this finding and to educate patients on the proper use of medication devices of their prescribed medications. The educational format with the greatest excessive demand is CMI, and this suggests that patients prefer to receive education through a mix of active and passive delivery formats. Standard CMI is not meant to be a stand-alone document, but an important tool that should be part of the interaction between health professionals and patients. Besides traditional education delivery formats, both patients and physicians would like to embrace other innovative forms of education, such as telephone consultation and medication database embedded within hospital information system. Effective use of these new forms of education format can offer convenience to both patients and physicians and complement to the traditional forms. Empowering patients with medication information through patient-centered delivery format can achieve an improved level of treatment adherence. ${ }^{11}$

The limitations of our study are the single center survey, and relatively small sample size in both surveys. For the patient survey, there might be some degree of selection bias in which only the younger and well-educated participants completed the survey. Because the study subjects were patients picking up prescriptions at the outpatient pharmacy, this may exclude elderly patients from participating in the study as caregivers often were the ones picking up the prescriptions for the older patients.

Patient medication education is now widely advocated in chronic disease management to increase medication adherence. Effective education requires the engagement of both patients and health care providers. Given that medication adherence is a complicated and multidimensional issue, research should be directed to identify current practice gaps and propose strategies for improvement. It is important to address patient-provider interaction during patient encounters as previous research has shown associations between communication and medication outcomes..$^{12,13}$

\section{Conclusion}

The study illustrates there is a mismatch between the information provided by health care providers, and the information desired by the patient during verbal counseling sessions. There is also a mismatch between the educational delivery formats provided to patients, and the desired forms preferred by patients and providers. These may act as barriers to conduct effective patient-centered education. Future education to patients should consider the current practice gap and explore ways to provide patient-centered tailored education content and delivery formats.

\section{Acknowledgment}

The authors thank Ting-Ting Qiu, who participated in this study.

\section{Disclosure}

The authors report no conflicts of interest in this work.

\section{References}

1. van Mil JW. Pharmaceutical care in community pharmacy: practice and research in Netherlands. Ann Pharmacother. 2005;39: 1720-1725.

2. Koo MM, Krass I, Aslani P. Factors influencing consumer use of written drug information. Ann Pharmacother. 2003;37:259-267.

3. Katz MG, Kripalani S, Weiss BD. Use of pictorial aids in medication instructions: a review of the literature. Am J Health Syst Pharm. 2008;63:2391-2397.

4. Smith MY, Wallace LS. Reducing drug self-injection errors: a randomized trial comparing a "standard" versus "plain language" version of patient instructions for use. Res Social Adm Pharm. 2013;9:621-625.

5. American College of Preventive Medicine. Medication Adherence Time Tool: Improving Health Outcomes. A Resource from the American College of Preventive Medicine. Washington, DC: American College of Preventive Medicine; 2011. Available from: http://www.acpm. org/?MedAdhereTTProviders. Accessed June 29, 2015.

6. Wright NJ, Greene JH, Wallston K, et al. Pilot study of a physiciandelivered education tool to increase patient knowledge about CKD. Am J Kidney Dis. 2013;62:23-32.

7. Kneeland PP, Fang MC. Current issues in patient adherence and persistence: focus on anticoagulants for the treatment and prevention of thromboembolism. Patient Prefer Adherence. 2010;4:51-60.

8. Denhaerynck K, Steiger J, Bock A, et al. Prevalence and risk factors of non-adherence with immunosuppressive medication in kideney transplant patients. Am J Transplant. 2007;7:108-116.

9. Van Dulmen S. The value of tailored communication for patientcentered outcomes. J Eval Clin Pract. 2011;17:381-383.

10. Lavorini $\mathrm{F}$. The challenge of delivering therapeutic aerosols to asthma patients. ISRN Allergy. 2013;2013:102418.

11. Moral RR, Torres LA, Ortega LP, et al. Effectiveness of motivational interviewing to improve therapeutic adherence in patients over 65 years old with chronic diseases: a cluster randomized clinical trial in primary care. Patient Educ Couns. 2015;98:977-983.

12. Taitel M, Jiang J, Rudkin K, et al. The impact of pharmacist face-to-face counseling to improve medication adherence among patients initiating statin therapy. Patient Prefer Adherence. 2012;6:323-329.

13. Zolnierek KB, Dimatteo MR. Physician communication and patient adherence to treatment: a meta-analysis. Med Care. 2009;47:826-834. 


\section{Publish your work in this journal}

Patient Preference and Adherence is an international, peer-reviewed, open access journal that focuses on the growing importance of patient preference and adherence throughout the therapeutic continuum. Patient satisfaction, acceptability, quality of life, compliance, persistence and their role in developing new therapeutic modalities and compounds to optimize

clinical outcomes for existing disease states are major areas of interest for the journal. This journal has been accepted for indexing on PubMed Central. The manuscript management system is completely online and includes a very quick and fair peer-review system, which is all easy to use. Visit http://www. dovepress.com/testimonials.php to read real quotes from published authors.

Submit your manuscript here: http://www.dovepress.com/patient-preference-and-adherence-journal 